Revista de Comunicación y Salud, 2018, Vol. 8, no 1, pp. 11-25

Editado por Cátedra de Comunicación y Salud

ISSN: 2173-1675

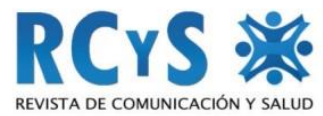

Enviado 23 octubre de 2017

Aprobado 7 de febrero de 2018

\title{
LA COMUNICACIÓN EN LA APS: UNA EXPERIENCIA MÁS ALLÁ DE LOS MEDIOS
}

\author{
Communication in the APS: an experience beyond the media
}

Johnny Alexander Gómez Granada

Facultad Nacional de Salud Pública - Universidad de Antioquia. Colombia

\begin{abstract}
Resumen
Como puede observarse, ambas estrategias tienen un fuerte enfoque conductista, centrado en modificar e influir en la conducta de las personas, puesto que conciben la comunicación como una herramienta o instrumento para lograr los cambios de comportamientos deseados (Mosquera, 2007). Además la débil reflexión práctica y teórica de la comunicación en el campo de la salud pública, promueve que las acciones que se realizan en el marco de estas estrategias tengan un enfoque informativo tradicional de comunicación, que se caracteriza por ser vertical, unidireccionales, impositivas y centradas en la difusión de conocimiento.
\end{abstract}

Palabras clave: salud, APS, comunicación, educación.

\section{Cómo citar el artículo}

Gómez Granada, J. A. (2018). La comunicación en la APS: una experiencia más allá de los medios. Revista de Comunicación y Salud, 8(1), pp. 11-25. DOI: http://doi.org/10.35669/revistadecomunicacionysalud.2018.8(1).11-25

\section{COMUNICACIÓN Y SALUD, UNA LARGA HISTORIA}

Hablar de la comunicación en el campo de la salud no es algo nuevo, es una relación de larga data, que se ha ido consolidando a lo largo de los años, gracias a la necesidad del personal de la salud de apoyarse en otras disciplinas, así como del interés de algunos comunicadores en los temas de bienestar. Son varias las conferencias y encuentros internacionales de salud, la mayoría promovidos por la OMS (Organización Mundial de la Salud), en los que se ha hablado del papel que cumple la comunicación en el campo sanitario, y principalmente en las apuestas que buscan la promoción de la salud y la prevención de la enfermedad. 
El primero de estos encuentros en hacer referencia a la comunicación fue el de Alma Ata en 1978, en el punto 7 de su conocida Declaración, en el que resalta que las acciones de Atención Primaria en Salud implican, además del sector sanitario, a todos los sectores relacionados y a diferentes aspectos del desarrollo nacional y comunitarios, entre estos las comunicaciones(Organización Mundial de la Salud, 1978). Ocho años después por medio de la Carta de Ottawa de 1986, se pone de relieve el papel de los medios de comunicación en la promoción de la salud y la necesidad de crear vías de comunicación entre el sector sanitario y los sectores sociales, políticos y económicos (Organización Mundial de la Salud, 1986).

De la misma forma en el encuentro de Santafé de Bogotá de 1992 sobre promoción de la salud, se habla de la importancia de la entrega de información y la promoción del conocimiento que constituyen valiosos instrumentos para la participación y los cambios de los estilos de vida en las comunidades (Organización Mundial de la Salud, 1992). Otro acuerdo internacional que se refiere a la comunicación en el campo de la salud, es la Carta del Caribe de 1993, en el que se menciona la responsabilidad que comparten las fuentes de información y los medios de educación y comunicación en el fortalecimiento de destrezas para el cuidado de la salud (Organización Mundial de la Salud, 1993).

En 1998 la Organización Panamericana de la Salud define las que deberían ser las Funciones Esenciales de la Salud Pública, dice la Función 3 que se titula Promoción de la Salud: "El desarrollo de acciones educativas y de comunicación social dirigidas a promover condiciones, modos de vida, comportamientos y ambientes saludables". (OPS/OMS, 1998, p. 68). Por último y para no extendernos más en esta contextualización internacional de comunicación y salud, la Declaración de Bangkok 2005 alude a las oportunidades que ofrece la globalización para mejorar la salud, en especial las relacionadas con las tecnologías de la información y la comunicación y los mecanismo disponibles para el intercambio de experiencias (Organización Mundial de la Salud, 2005).

Como lo vemos hasta aquí son múltiples las referencias internacionales que hacen alusión a la comunicación en los contextos de la salud, en la mayoría de ellas está presente una marcada tendencia a reducirla a los medios de información o sobre el papel que cumple la comunicación en la difusión de información sanitaria y la comunicación entre los actores de los sistemas de salud.

En el contexto nacional mientras tanto, son pocas las disposiciones que tiene en cuenta la comunicación como un componente importante en el campo de la salud, resaltamos dos por su trascendencia y actualidad: el Plan Decenal de Salud Pública (MSPS - PDSP, 2012) que dentro de las estrategias resalta la Información, Educación y comunicación (IEC) como un componente importante para su implementación; e igualmente la recientemente sancionada Política de Intención Integral en Salud (Ministerio de Salud y Protección Social, 2016), que reconoce como una dificultad la poca formación del talento humano en salud en comunicación y la necesidad del fortalecimiento de la comunicación entre los diferentes actores del sistema. 


\section{2. ¿DOS PARADIGMAS ENFRENTADOS O COMPLEMENTARIOS?}

En lo que se refiere a la comunicación básicamente se pueden identificar dos grandes paradigmas sociales: el difusionista-informativo y el relacional-dialógico; un paradigma social según Capra (1996) es una "constelación de términos, valores, percepciones y prácticas que tienen en común una comunidad y que forma una determinada vista, percepción de la realidad que es el substrato de la manera como esta sociedad se auto-organiza" (Citado en Gonzáles, 2005, p. 32). Es importante mencionar que estos sentidos dados, según el paradigma, atraviesan todos los subcampos de especialización o de acción de la comunicación, como es el educativo, el organizacional y por supuesto, la salud.

Son estos dos lentes los que definen a grandes rasgos el pensar y el hacer de la comunicación desde mediados del siglo pasado hasta la actualidad. Veamos a continuación una tabla comparativa, para comprender cada uno de ellos, e identificar sus características, fortalezas, limitantes y cualidades:

\section{Tabla 1.}

Comparativo paradigmas de la comunicación. Adaptado de Gumucio $^{1}$ del ensayo Cuando el doctor no sabe (Gumucio Dagron, 2010).

\begin{tabular}{|l|l|}
\hline \multicolumn{1}{|c|}{ Difusionista - Informativo } & \multicolumn{1}{c|}{ Relacional - Dialógico } \\
\hline- Problema: falta de información, & - Problema: inequidades sociales \\
\hline - Vertical & - Horizontal \\
\hline- Persuasión & - Concienciación - emancipación \\
\hline- Pasiva y "bancaria" & - Activa y crítica \\
\hline- Cambios individuales & - Cambios colectivos \\
\hline- Objetos de cambio & - Agentes de cambio \\
\hline- Difusión masiva & - Dialogo y debate - interpersonal \\
\hline- Diagnósticos generalizados & - Diagnósticos específicos \\
\hline- Corto plazo & - Largo y mediano plazo \\
\hline- Mensajes - campaña - resultados & - Procesos \\
\hline- Acceso controlado & - Apropiación en toma de decisiones \\
\hline- Diseminación de información por medios & - Participación comunitaria, interacción \\
\hline masivos & \\
\hline- Prioridades de los patrocinadores & - Necesidades de la comunidad \\
\hline
\end{tabular}

El paradigma difusionista es heredero de las corrientes de pensamiento positivistas, modernistas y conductistas, "se constituyó como el paradigma hegemónico según el cual el proceso de comunicación se establecía de manera unidireccional a partir dela relación estímulo/respuesta, centrándose en los procesos de transmisión y recepción de

\footnotetext{
1 Alfonso Gumucio Dagron: Escritor de Bolivia, cineasta, periodista, fotógrafo y especialista en comunicación para el desarrollo. Es autor de varios libros sobre cine y comunicación para el cambio social, así como documentales, exposiciones fotográficas y cientos de artículos en revistas.
} 
información" (Pinto, M. C., Jiménez García, 2016, p. 13).Visión que todavía hoy domina en los diferentes ámbitos de la comunicación, incluido el sanitario.

El paradigma relacional mientras tanto surge en los años 60 del siglo pasado como una alternativa al tradicional, empujado por las Teorías de la Dependencia, promulgadas por diferentes científicos sociales vinculados a la Comisión Económica para América Latina y el Caribe (CEPAL). En Suramérica ha tenido dos momentos, en un principio se conoció como comunicación para el desarrollo, pero después de los 80 , gracias a los cuestionamientos de diferentes pensadores y educadores latinoamericanos que cuestionaban su marcado institucionalismo, tecnicismo y verticalidad sobre el saber local, fue sustituido por la comunicación para el cambio social; esta última toma elementos de la comunicación interpersonal y de la educación popular latinoamericana, en el que se privilegia los procesos comunicativos por encima de los productos (A., 2015).

Consideramos que más que paradigmas enfrentados, son ante todo complementarios, la dificultad radica en que como el difusionista es hegemónico siempre existe una tendencia a reproducirlo sin mayor dificultad; es necesario hacer el esfuerzo y volverlo consciente para superarlo y poder trascender a una comunicación más transformadora e incluyente. Ambos paradigmas son necesarios en el campo de la salud, todo depende de los objetivos que se persiguen y la intencionalidad que se tenga.

Una parte fundamental a tener en cuenta es que en cada uno de ellos subyace una forma de ver al otro y a la comunidad, el relacional dialógico concibe al otro como un ser igual, con las mismas capacidades y falencias que cualquier otro, es una visión más humana de la sociedad, "la comunicación para el cambio social o desarrollo, emerge como respuesta a la indiferencia y al olvido, rescatando lo más valioso del pensamiento humanista que enriquece la teoría de la comunicación"(A., 2015).

El proyecto APS Suroeste le ha apostado por tener una comunicación equilibrada en estos dos paradigmas y de no caer en una visión dicotómica, de idealizar uno de los dos; los procesos comunicativos son complejos y requieren de todas las herramientas y conocimiento que cada uno de los paradigmas ha podido desarrollar y perfeccionar a lo largo de los años. Por ejemplo, se considera que el informativo es necesario en todo momento, pero en especial al inicio, para el reconocimiento de la estrategia APS, el posicionamiento del proyecto y la trasmisión de mensajes de manera rápida, también cómo negarse al aprovechamiento de los diferentes recursos de difusión que ofrecen las Tecnologías de la Información y la Comunicación, y los medios de información locales de cada uno de los municipios.

Hay que dejar claro que históricamente la comunicación que se ha realizado en el campo de salud colombiano, es la informativa, ya que esta le calza muy bien a un sistema de salud orientado hacia lo morbicéntrico, centrado en la tecnología, la verticalidad, el conocimiento técnico y el mercado. 
La comunicación para el cambio social, por su lado es indispensable para apuestas que buscan transformarlas realidades de las comunidades, se ha desarrollado principalmente en los movimiento sociales, la participación popular, proyectos de ONG y de educación popular; es una comunicación que exige el involucramiento de sus actores, que demanda las relaciones de igual a igual y la participación de las comunidades en la toma de decisiones. Como lo dijimos más arriba, si para el modelo de salud hospital o centrista hegemónico, la comunicación difusionista le calza muy bien, para una estrategia como la Atención Primaria en Salud, este enfoque comunicativo se queda corto. Al cambiar la manera de gestionar la salud de una forma asistencial, a un modelo centrado en la APS, que busca la promoción de la salud, centrada en las personas, es integral, promueve la participación comunitaria y su autorresponsabilidad, la intersectorialidad y la educación (Organización Mundial de la Salud, 1978); los enfoques de comunicación también deben cambiar.

\section{LOS ESCENARIOS DE LA COMUNICACIÓN EN LA SALUD: LA APS}

Son múltiples los escenarios dentro del campo de la salud donde la comunicación ha podido encontrar donde desarrollarse, algunos de ellos tiene que ver con el periodismos e información en salud; la comunicación organizacional en instituciones de salud; la comunicación interna en equipos de salud; publicidad y marketing de servicios de salud o social; comunicación del riego; E-salud o Tecnologías de la Información y la Comunicación TIC; comunicación interpersonal médico - paciente; comunicación y educación para la salud; participación o movilización social en salud, comunicación pública; comunicación comunitaria o popular, entre otras.

Todos estos campos de acción contribuyen a incrementar el conocimiento de la comunicación en la salud, no existen mejores o peores, cada uno aporta desde su especialidad e intereses, algunos con posturas más humanas que otras, otros defendiendo intereses institucionales o de mercado, otros en cambio con un gran compromiso con las comunidades; esto dependiendo del paradigma en el que se paren y las lentes con que ven el mundo. Tampoco sucede que uno reemplace a otro, todos conviven y encuentran su nicho de acción específico.

De estos escenarios de acción, vemos que los cuatro últimos son los que tiene mayor relación con la comunicación para el cambio social, o que se enmarcan en este enfoque, (comunicación y educación para la salud; participación o movilización social en salud, comunicación pública; comunicación comunitaria o popular). También podemos observar que estos mismos a la vez, siguen unos principios similares a los de la estrategia de APS.

Podemos entonces decir que una estrategia de APS se puede servir de los diferentes paradigmas de la comunicación, así como de sus distintos campos de acción, pero que definitivamente su apuesta central en comunicación debe ir orientada a la comunicación para el cambios social, es con la que comparte mayor afinidad y tienen unos objetivos y compromisos sociales, que las hace indispensables la una de la otra. 


\section{DEL DICHO AL HECHO, GRAN TRECHO}

Pero implementar una apuesta de comunicación para el cambio social en el campo de salud no es tarea sencilla, y casi en cualquier campo, ya que como lo hemos dicho el paradigma hegemónico es el difusionista y siempre nos está empujando hacia él; para poner este ejemplo en el contexto de salud, pasa lo mismo con la estrategia de APS, que pretende romper la forma de gestionar la salud en los territorios, pero se encuentra con que toda la infraestructura, tanto física, logística, de talento humano, cultural y financiera, están volcadas hacia el modelo asistencialista que es el hegemónico; por lo que su implementación requiere de un gran esfuerzo adicional y de la voluntad y convencimiento de los diferentes actores del sistema, para no seguir reproduciendo los enfoques tradicionales.

Dentro de las razones o dificultades que normalmente se encuentra para virar hacia la comunicación para el cambio social en salud, primero que todo estamos los propios profesionales, tanto comunicadores, que nos hemos formado en escuelas de comunicación que continúan impartiendo los mismos conocimiento de hace 50 años, y el personal de la salud, que carecen de toda formación pedagógica; segundo la instrumentalización de la comunicación y las expectativas de los actores del sistema; y por último está la apatía de las comunidades para participar, que en muchos casos solo lo hacen cuando obtienen algo a cambio, y los que no, han tenido experiencias de participación que solo los utilizan para la foto o incluir en los indicadores.

\section{DEFORMACIONES Y DE ESCUELAS DE FORMACIÓN}

Si hay algo claro, es que las estrategias de comunicaciones y las de salud como la APS, son desarrolladas por seres humanos que se forman, en la mayoría de los casos, para cumplir con esta misión, son los encargados de planear y ejecutar las acciones en estas áreas del conocimiento; los primeros son profesionales de la comunicación que cursan en las facultades, por varios semestre, una gran cantidad de materias que se supone los preparan para enfrentarse a la vida laboral; los segundo mientras tanto son profesionales de la salud, que en sus diferentes carreras y niveles de formación, se preparan para cumplir su rol y responsabilidad dentro del sistema de salud.

Las facultades de comunicación han tenido poca transformaciones curriculares en los últimos años, basta con revisar el pensum de una de las más reconocidas facultades de nuestro departamento, para constatar que siguen ancladas al paradigma difusionista de la comunicación, en el que se privilegia la técnica y el dominio del instrumento, sobre el pensamiento estratégico; en el que se idealizan los medios masivos de información, como la televisión, la radio y la web por encima de los procesos sociales; en el que las materias son sobre imagen gráfica, diseño, redacción, fotografía, periodismo, relaciones públicas y solo una de comunicación para el desarrollo.

Es decir, que los actuales egresados de comunicación, salen experto en manipular equipos, redactar noticias, presentar eventos, pero con unas marcadas falencias para desarrollar proceso de comunicación con las comunidades, así como ausencia en 
formación pedagógica, como la escucha, tan necesaria en la comunicación para el cambio social; actualmente hay una marcada necesidad, en proyectos sociales y de salud, que ha hecho que más que la academia, sea la práctica la que termine de formar a este tipo de comunicador, que Gumucio llama el "comunicador/educador" (Gumucio Dagron, 2010, p. 83), "que lo esencial de su tarea no consiste en producir mensajes y paquetes educativos tanto como de hacer posible un proceso de participación y educación cuyo eje es el diálogo de saberes y la acción colectiva".

Otras características que debe comprender este comunicador es que:

...el proceso de comunicación con la gente y dentro de las comunidades es más importante que las ayudas impresas o audio-visuales que puedan surgir de esa dinámica. Es en el proceso de comunicación y participación que se inicia el cambio social. El nuevo comunicador debe comprender que la tecnología es una herramienta y nada más que una herramienta. La tecnología puede apoyar el proceso de comunicación, pero este no puede depender totalmente de ella. (Gumucio Dagron, 2010, p. 83).

Una pregunta que nos planteamos acá es, ¿cómo puede un comunicador aplicar la comunicación dialógica, si la mayoría del tiempo lo está usando para generar contenidos y está atendiendo a las demandas y expectativas que tiene los profesionales de la salud y los jefes, sobre la elaboración de materiales?

En cuanto al personal de la salud, tanto profesional como técnico, el panorama no es más alentador, las escuelas de medicina, enfermería, odontología, nutrición y psicología, entre otras, dotan a los estudiantes sobre conocimiento técnicos en enfermedades, tratamientos, procedimientos, diagnósticos, equipos, prevención, medicamentos; y no sobre comunicación, que puede ser comprensible, pero que lo dejen de hacer en herramientas pedagogía y en estrategias para el trabajo con comunidades, es una falencia preocupante para los proyectos de promoción de la salud y que tienen una base social, que en muchos casos no encuentran profesionales con la experiencia, sensibilidad y habilidad para el trabajo con comunidades.

Algo que ha ocasionado este fenómeno es la tendencia a la hiperespecialización de los profesionales de la salud, que en los últimos años va en aumento, tanto que en la Política de Atención Integral en Salud (2016), en el análisis del contexto institucional, se reconoce esta situación:

La formación de talento humano en salud ha respondido a estas realidades, lo cual ha conllevado a la pérdida progresiva de competencias de algunas profesiones en áreas como salud pública, comunicación, gestión, comprensión del contexto, humanización y calidez en la atención y, en algunos casos, en las áreas clínicas. Se agrega que durante los años posteriores a la reforma no se dio la formación de competencias asociadas a la educación y promoción de la salud, APS, procesos de gestión y coordinación de programas e intervenciones de carácter familiar y comunitario.(Ministerio de Salud y Protección Social, 2016, p. 20). 
Si el país ha seleccionado la estrategia de APS como la encargada para gestionar la salud en todo el territorio nacional, y así lo corroboran las últimas disposiciones legales, se hace necesario que las diferentes instituciones encargadas de la formación del personal de la salud y sociales, replanteen los currículos académicos y los perfiles de los profesionales que están formando, para poder responder a este compromiso con la salud de los colombianos.

\section{DESINSTRUMENTALIZAR LA COMUNICACIÓN: PASAR DEL PRODUCTO AL PROCESO}

La comunicación debe ser una estrategia que permita el entendimiento y la comprensión entre sujetos para llegar a posteriores acuerdos que beneficiarán a toda una comunidad. Algunos elementos asociados a las comunicaciones han sido la televisión, radio, prensa y redes sociales. Para el común de las personas, comunicar se limita exclusivamente a enviar un mensaje e informar; desconociendo lo esencial de esta, que es el diálogo y la puesta en común de ideas que pretenden cambiar o mejorar una realidad social.

La percepción tradicional de las comunicaciones instrumentales y operativas, donde los medios difusores de mensajes son el epicentro del funcionamiento de los procesos sociales, donde la cantidad siempre está superada por la calidad, en el que es más importante mostrar el producto que el mismo proceso como tal; debe ser cambiada por la concepción que, antes de hacer comunicaciones es necesario conocer el territorio, las personas que lo habitan, sus costumbres y cultura, los medios más efectivos para brindarles información y las necesidades sociales básicas insatisfechas que realmente tienen. Además, comprender la importancia de una labor cumplida responsablemente y fundamentada en el profesionalismo y no en el placer de mostrar resultados, que de una u otra forma, no demuestran el éxito en los procesos desarrollados para lograr los mismos.

Normalmente se cree que los carteles, los mensajes radiales y algunos comerciales televisivos suple la necesidad de ir hasta las comunidades y de conocerlas; por esto, muchos procesos comunicacionales no funcionan, se intenta trabajar con comunidades que no conocemos, todo un error a la hora de ejecutar actividades en colectivos poblacionales.

Otro paradigma que se tiene dentro de las comunicaciones trabajadas en los ámbitos comunitarios, es la asociación de un "buen trabajo" de acuerdo al elevado número de asistentes o participantes en una actividad. En la mayoría de los casos, trabajar con grupos reducidos permite un mayor acercamiento de los participantes a los temas tratados o trabajados, un mejor entendimiento, una participación más activa y la posibilidad de brindar explicaciones personalizadas; en pocas palabras, puede ser más efectivo trabajar con grupos reducidos si lo que se busca es educar y comunicar.

La necesidad de ver resultados exitosos en poco tiempo también se convierte en un limitante dentro de las comunicaciones. El trabajo con comunidades no permite obtener

Revista de Comunicación y Salud, 2018, Vol. 8, oㅜ 1, pp. 11-25 
logros en tan sólo algunos días, se requiere de tiempo para conocer la cultura, dificultades y necesidades de las personas que habitan un territorio, también se requiere tiempo para educar y generar cambios de hábitos; por lo tanto, los procesos comunicativos dependen en gran medida del tiempo destinado para lograrlos.

Teniendo en cuenta el panorama anterior como fundamento conceptual de la comunicación, es importante mencionar que al hacerse un análisis del tema en las distintas secretarías de los municipios de la cuenca del río San Juan, el horizonte encontrado deja ver que la concepción del término comunicación es entendido como un método para mostrar resultados y abarcar grandes públicos; y no como una estrategia que posibilitará la educación, logrando un mayor éxito dentro de las actividades propuestas. Para ellos, inicialmente, comunicar era sólo: pegar un afiche, publicar notas radiales y televisivas y utilizar las redes sociales como canal de información más seguro para llegar a un público vasto; y no veían la comunicación como una forma de ir hasta las comunidades, de conocerlas y de contribuir en su desarrollo social, por ello, es importante entender que los medios de difusión permiten informar y por esto cumplen una labor importante dentro de la comunicación, pero esta debe de ir más allá, el diálogo con las comunidades debe ser el centro.

En la actualidad, la concepción del concepto de comunicación en las distintas secretarías, y hasta en las comunidades con que se ha tenido contacto, ha cambiado, ya le ven la importancia de esta dentro de los procesos sociales, ya no se habla de informar sino de dialogar, se le da más importancia al proceso y la calidad, que al producto y la cantidad. Aún quedan muchos conocimientos por aprender, pero se ha reflejado el cambio y se han visto los primeros resultados de esta apuesta de aproximarnos a desarrollar un proceso de comunicación para el cambio social en los municipios del proyecto.

\section{PODEMOS COMUNICAR SI DAMOS PIE A LA PARTICIPACIÓN}

Partiendo que la palabra comunicación proviene del latín communicatio que significa compartir algo o poner en común, y que comparte la misma raíz con comunidad (Real Academia Española, 2017); la interacción con el otro y la comunidad es una necesidad, promover que a los actores participen para identificar sus verdaderas necesidades es una premisa. Tenemos que interactuar con las personas, hablar, darles la voz, eso es más importante que grabar comerciales, hacer pendones o subir fotografías a las redes sociales. $Y$ esto debe hacerse con cada uno de los actores que participan en los procesos de salud.

Aquí un ejemplo de algunas de las experiencias vividas en el proyecto BUPPE APS Suroeste. ¿A alguien se le ha ocurrido poner a escribir a una promotora de salud?, pues algunas del municipio de Hispania en Antioquia lo hicieron. Allí, una de ellas da cuenta de la forma como vive una familia, claramente con problemas de convivencia e insatisfechos con la forma en la que opera la estrategia. Su texto, después de narrar algunas discusiones entre los habitantes de la casa, concluye con una frase del cabeza del hogar. "La próxima vez vuelva con ayudas". 
El relato, aunque duro pero emotivo, revela dos situaciones. Una es que podemos identificar necesidades simplemente escuchando y observando comportamientos en las familias, más allá de llenar una ficha densa y repetitiva. Y la última situación es que las familias están cansadas de recibir este tipo de visitas, esto deja ver además que ir a tomar la presión arterial y hacer demanda inducida no es hacer APS, eso no resuelve el problema de convivencia familiar que tenemos en esa familia, por ejemplo. Estamos dándoles a las comunidades de lo mismo, partiendo del supuesto que esas son sus necesidades sin antes escucharlos y analizar su situación.

¿Se han puesto a pensar qué podría contar un niño, una madre de familia a través de una historia escrita? ¿Qué podrá decirnos un joven en una canción que él mismo componga, o en un poema, cuento o un dibujo? No sabemos, porque nos dedicamos a hacer diagnósticos con herramientas estandarizadas y repetitivas, ¿por qué es más importante cuantificar y difundir que intervenir de forma estratégica y creativa?

La comunicación, orientada hacia el cambio social es capaz de mostrar resultados exitosos en las comunidades, y esto ni siquiera tiene que estar en las teorías, es un asunto de simple lógica entender que cuando hay relación con las personas; si se está frente a frente con ellas para conocer sus historias, sus modos de relacionarse con los demás, su entorno en general, será más probable hacer un proceso efectivo en sus territorios.

Son muy bonitas las fotografías, posters y demás publicaciones que vemos diario en las redes sociales y páginas web de las instituciones; vemos cómo "calman el hambre" de algunas personas y sacan pecho publicado tales actividades, pero la jornada termina, el hambre regresa y los problemas sanitarios continúan, la organización comunitaria permanece débil, aumentan los problemas de contaminación, drogas, convivencia y todos aquellos que se nos ocurran, pero están ocultos porque no se tomó el trabajo de hacer diagnósticos participativos con la comunidad, y es que talvez las autoridades no se atreven asacar estas realidades a la luz porque no hay proyectos ni programas para atenderlos.

Los actores institucionales, como lo son las promotoras de salud, están sujetas a cumplir unas metas que se fijan desde el inicio de año; algo difícil de calcular sin saber a qué poblaciones se van a enfrentar, si una de las quejas de ellas mismas es que hay poblaciones migrantes que imposibilitan la continuidad en los procesos que se emprenden. Sin contar con que no tienen en cuenta en sus planes los tiempos de cosecha cafetera donde todos los que se dedican a esta actividad agrícola están en función de ello en determinado tiempo del año, y esto es esencialmente un problema de comunicación, la simple ausencia de escuchar al otro y planear en consecuencia.

Lo anterior debe llevarnos a pensar entonces en que falta una planificación mejor pensada para hacer APS de forma acertada; y la forma no es otra distinta que trabajar por el bienestar conjunto de las localidades y no por el afán de mostrar cifras de "población atendida". Si seguimos trabajando así lo único que lograremos es que las 
personas sigan sintiéndose beneficiarios dentro del sistema de salud paternalista y no como actores activos de sus propios cambios.

Si logramos darle participación a las personas, apoyándonos en el conocimiento que tienen sobre sus barrios, veredas y organizaciones podremos empoderarlos de sus realidades, hacerles entender que ellos desde su accionar como comunidad organizada pueden dar fe de sus verdaderas necesidades y contribuir a la solución. Nunca les hemos dicho a las personas que son ellas las encargadas de llevar la salud a sus lugares de residencia. Nos preocupamos por satisfacer lo que nosotros consideramos una necesidad y terminamos siendo asistencialistas y no dinamizadores de procesos. La población no se incluye únicamente dando atención, hay que darles participación en la toma de decisiones.

\section{LOS RETOS DE LAS ESTRATEGIAS DE COMUNICACIÓN EN SALUD}

Alguna vez el comunicólogo Gumucio Dagron (2010) mencionó que por años ha visto en el área de la salud "acciones de "comunicación para el desarrollo" que no comunican"(p. 69); también dijo que una de las posibles causas es la confusión entre comunicación e información, que "contribuyen a distorsionar aún más el escenario, impidiendo la formulación de verdaderas políticas y estrategias de comunicación, $y$ sustituyendo a estas con campañas puntuales y un activismo que ya han demostrado con creces su ineficiencia" (p. 74). Esto puede ser tomado como una invitación a reflexionar sobre el porqué, en muchas ocasiones, las estrategias de comunicación no logran los resultados esperados.

Por lo general las disciplinas de la comunicación y la educación en el campo de la salud se han abordado de forma aislada y desarticulada; en los últimos años se ha presentado un auge de propuestas que pretenden integrar estas dos disciplinas pero no han tenido los resultados esperados. Una de la más conocida y utilizadas en el contexto latinoamericano es la estrategia I.E.C (Información, Educación y Comunicación), la cual busca "promover o consolidar, a través de la combinación de tecnologías, enfoques comunicacionales y procesos de socialización; cambios de comportamiento o actitudes en determinadas audiencias en áreas del desarrollo como salud y educación" (Mocelli, n.d.).

Otra estrategia frecuentemente usada en diferentes programas de salud es la estrategia COMBI (Communication for Behavioral Impact - Comunicación para impactar conductas) con la que se pretende de una manera "estratégica y estructurada, planificar, poner en práctica y vigilar la movilización y comunicación social, para lograr y mantener resultados conductuales muy específicos relacionados con la prevención y el control de las enfermedades transmisibles" (Lloyd, 2005).

Como puede verse, ambas estrategias tiene una fuerte apuesta conductista centradas en modificar e influir en los comportamientos de las personas, en las que se privilegia el componente informativo y las concepciones que tiene de educación y comunicación son limitadas, se soportan desde un modelo tradicional de educación, y la

Revista de Comunicación y Salud, 2018, Vol. 8, nº 1, pp. 11-25 
comunicación mientras tanto se desarrolla con una perspectiva difusionista, centrada en los medios masivos y tecnológicos.

Sin duda un reto grande para las comunicaciones es, y ha sido, trascender precisamente esa brecha de lo meramente informativo, sin negar que los medios y los productos de comunicación son valiosas herramientas de difusión, pero el contexto las ha confundido como las comunicaciones mismas, distorsionando lo que son en esencia: un proceso de interacción. Y es que ésta es una de las potenciales causas que pueden llevar a que muchas estrategias de comunicación no logren sus objetivos, porque están centradas únicamente en el producto, en la necesidad de mostrar resultados para cumplir con determinados indicadores, sin importar el impacto real que puedan tener.

A lo anterior muchas veces se suma la homogenización de las audiencias y una idealización del alcance de los medios de información. Una estrategia de comunicaciones que busque, por ejemplo, la promoción de temas de la salud a través de los medios, requiere de una lectura de las realidades sociales y las dinámicas comunicacionales del territorio para determinar si es pertinente 0 no; entonces es necesario cuestionarse sobre: ¿con qué comunidad quiero trabajar?, ¿el medio que pienso utilizar llega hasta su hogar?, ¿qué lenguaje debería usar?, ¿cómo hacer para que el tema les interese?, ¿con qué recursos, tanto técnicos como humanos cuento para llevar a cabo la estrategia?, etc.

En los municipios que participan en el proyecto APS Suroeste, se pudo evidenciar las notables diferencias comunicacionales en cada uno de los territorios: mientras que en unos abundan los medios locales, con audiencias frecuentes y un alcance de casi todo el territorio, otros carecían de estos, no contaban con profesionales y recursos técnicos que apoyaran la realización frecuente de programas, o tenían un alcance limitado a lo urbano y a una porción del área rural a la que llega la señal. En esta perspectiva, pensar solamente en el producto no es garantía de que la estrategia cumpla el objetivo propuesto, pues se ha evidenciado que la efectividad de los programas y las campañas de comunicación para la salud, dependen de la participación activa de la comunidad que, directa o indirectamente, son afectadas en la implementación de las estrategias de prevención de la enfermedad y promoción dela salud, en las que es fundamental que se tenga en cuenta su contexto social.

Finalmente es necesario reiterar que los medios de información y los productos no son la hermanastra fea, rencorosa y malintencionada que hacen imposible la vida de la cenicienta de las comunicaciones; de hecho son herramientas bastante valiosas cuando se utilizan como apoyo a los procesos, como parte de las estrategias y como un puente para promover la participación e inclusión de las comunidades en las acciones que finalmente los beneficiarán. La fotografía, por ejemplo, más que una técnica para capturar imágenes a través de una cámara, puede convertirse en una herramienta para reconocer y retratar la realidad sobre un tema o una problemática de interés para una comunidad, que adquiere un valor agregado cuando estas mismas pueden participar activamente en el proceso. Aun así, es pertinente mencionar que los procesos de comunicaciones, como todos los procesos en las diferentes áreas del conocimiento, 
también requieren de recursos tanto técnicos como humanos para alcanzar sus resultados, y esto es un aspecto que a veces se olvida en las estrategias de comunicación.

\section{ALGUNAS REFLEXIONES SOBRE LA APS}

\subsection{Trabajo intersectorial}

Como se ha dicho, trabajar con salud implica involucrar diversos actores de la sociedad si queremos lograr un alto impacto en la intervención del problema. Una problemática de drogadicción en jóvenes por ejemplo, que afecta la convivencia en un sector, la salud mental de las familias e impide el desarrollo de las poblaciones, debe trabajarse con todas los actores que allí convergen.

Ahí tiene responsabilidad secretaría de educación, son ellas las responsables de velar por la educación y orientación de los menores, brindar programas educativos que fortalezcan los entornos escolares y comunitarios. Las comisarías de familia deben hacer seguimiento, vigilancia o control en la forma que los niños, niñas y adolescentes están siendo atendidos por sus padres. La policía nacional es la encargada de inspeccionar el expendio de sustancias psicoactivas.

Como ven, en una sola problemática que está directamente relacionada con la salud de las persona ya se han identificado tres instituciones; y por ningún lado aparecen las secretarías de salud, esto no quiere decir que no deban participar; la atención debe centrarse en el hecho de que no son solamente las secretarías de salud son las encargadas de atender tales situaciones. Sin contar que la participación de la comunidad, de líderes comunitarios o actores sociales será clave para identificar las posibles causas por las que se presenta el consumo en ese sector. La salud es un asunto de todos, y todos debemos trabajar de forma articulada para la resolución de conflictos en la sociedad.

\subsection{Atención de acuerdo a la realidad}

Una queja constante de los equipos básicos de salud es la escasa participación de algunos sectores en cualquier capacitación. Mientras que algunos son receptivos a las brigadas de salud y programas de escuelas saludables otros son totalmente reacios a participar. Sin embargo quienes participan siempre reciben más de lo mismo y la deserción en las actividades empieza a crecer.

Con este panorama la solución es simple. Quienes son receptivos deben empezar a recibir otro tipo de formación, no solo recibir charlas que ya se saben de memoria sino que deben empezar a escuchar otros discursos desde los determinantes sociales de la salud; estas comunidades ya están preparadas para conocer en mediana medida cómo funciona nuestro sistema de salud; esas localidades están listas para formarse como actores en temas de salud. Si empezamos capacitarlos desde ahí, empezarán a 
descubrir otro mundo detrás de la salud y verán que ellos pueden hacer mucho desde sus territorios para empoderarse de sus procesos.

Entre tanto, en aquellas poblaciones que no participan, basta con identificar un pequeño grupo organizado, junta de acción comunal, grupo de tercera edad, grupo juvenil etc. y empezar a llegarle a esa comunidad desde esos grupos que en últimas son los legitimadores en ese territorio. No hay que pensar en cubrir un grupo numeroso de personas, lo importante es instalarse en una comunidad, picar una pequeña parte y que sean ellos los encargados de contagiar al resto. Esto se logra haciendo diagnósticos participativos.

\section{BIBLIOGRAFÍA}

A., G. (2015). Comunicación para el Cambio Social. Recuperado de http://www.comunicacionparaeldesarrollo.org/media/uploads/cyclope_old/adjuntos/co municacin-para-el-cambio-social-120250138865361-4.ppt

Gonzáles, F. (2005) ¿Qué Es Un Paradigma? Análisis Teórico, Conceptual. Investigación y Postgrado, 20(1), 13-54.

Gumucio Dagron, A. (2010). Cuando el doctor no sabe. Estudios Sobre Las Culturas Contemporáneas, Epoca II, 67-93.

Lloyd, L. S. (2005). COMBI: Comunicación para impactar conductas. Recuperado de http://www.ops.org.bo/textocompleto/iden28034.pdf

Ministerio de Salud y Protección Social. Política de Atención Integral en Salud (2016).

Recuperado de

https://www.minsalud.gov.co/sites/rid/Lists/BibliotecaDigital/RIDE/DE/modelo-pais-

2016.pdf

Mocelli, M. (n.d.). Estrategias de información, educación y comunicación: objetivos, modelos y teorías. Recuperado de

https://www.google.com.co/url?sa=t\&rct=j\&q=\&esrc=s\&source=web\& $c d=1 \& c a d=r j a \& u$ act=8\&ved=0CBwQFjAAahUKEwiOIMymmo7HAhUDbR4KHVITBs4\&url=http://www.o sancolombia.gov.co/Portals/0/TalleresEstrategialEC/2 Estrategias de Información, Educación y

MSPS - PDSP. (2012). Plan Decenal de Salud Pública, PDSP, 2012 - 2021. Ministerio de Salud. http://doi.org/10.1177/1757975912453861

OPS/OMS. (1998). La Salud Pública en las Américas. Nuevos conceptos, análisis de desempeño y bases para la acción. Publicación Cientifica y Técnica, 589, 1(OPS), 417. Recuperado de http://www.paho.org/hq/index.php?option

Organización Mundial de la Salud. (1978). Declaracion de Alma-Ata. In Conferencia Internacional sobre Atención Primaria de Salud, Alma-Ata, URSS, 6-12 de septiembre de 1978 (p. 3). Alma-Ata. Recuperado de http://www.promocion.salud.gob.mx/dgps/descargas1/promocion/1_declaracion_deA LMA_ATA.pdf

Organización Mundial de la Salud. (1986). Carta de Ottawa para la promoción de la salud. Promocion De La Salud. Recuperado de http://www.promocion.salud.gob.mx/dgps/descargas1/promocion/2_carta_de_ottawa. pdf 
Organización Mundial de la Salud. (1992). Declaración de la Conferencia Internacional sobre Promoción de la Salud. Promoción de la Salud en América Latina (p. 4). Santa $\mathrm{Fe}$ de Bogotá. Recuperado de http://www.bvsde.paho.org/bvsacd/cd51/promocion-bogota.pdf

Organización Mundial de la Salud. (1993). Carta del Caribe. In Carta del Caribe (p. 5). Puerto España.

Organización Mundial de la Salud. (2005). Carta de Bangkok para la promoción de la salud en un mundo globalizado (pp. 1-6). Recuperado de http://www.who.int/healthpromotion/conferences/6gchp/BCHP_es.pdf

Pinto, M. C., y Jiménez García, L. (2016). Estado de la cuestión en comunicación para el cambio. In Comunicación para la movilización y el cambio social (p. 191). Medellín: Universidad de Medellín.

Real Academia Española (2017). Recuperado de http://dle.rae.es/?id=A58xn3c 